\title{
Analysis of the "Harmony" Education Ideas in Chinese Taijiquan
}

\author{
Shiliang Yan \\ Institute of Physical Education, Dezhou University, Dezhou 253023, China \\ Tel: 86-534-898-5157Ｅ-mail: tyxys1@163.com
}

Received: November 22, 2010 Accepted: December 2, 2010 doi:10.5539/ies.v4n3p133

\begin{abstract}
In this article, many methods such as the literature study are used to analyze the harmony ideas in the sport, the education, and the development of Taijiquan, and the deep meanings contained in these three aspects are expounded in detail, and the conclusion indicates that Taijiquan is very important both for the public body-building and for the harmonious development of the society.
\end{abstract}

Keywords: China, Taijiquan, Harmony

\section{Introduction}

As the important part of the Chinese Martial Arts, Taijiquan has been influenced and interiorized by the traditional culture of china in past three hundreds years, so it has deep cultural meaning and spirit values. In the harmonious times, it is very helpful to deeply understand its connotations and expand its exercise group for the advancement of the human society. From the sports, the education, and the development of Taijiquan, the harmony education concepts will be expounded as follows.

\section{Harmony in Sports}

Taijiquan is not only a kind of boxing, but also a kind of sports. As viewed from human sports, the visible is the formation of sports, and the invisible is the meaning of sports, i.e. the practitioners' heart, so both from the heart mood and from the technical expression, Taijiquan would present "the beauty of harmony".

\subsection{Harmony in the Exterior}

"The beauty of harmony" in the tradition of China is embodied in various aspects of the social life, either in Taijiquan. In the technical representation, it opposes intolerance, and emphasizes the integration of different and opposite factors to form harmonious and uniform unity (Wang, 2009, p.64-67).

The first block of Taijiquan is full of symmetric and harmonious forms because of its formative arts, actions with alternate kindness and severity, harmonious opening and closing, visible and invisible exchanges, proper speed, loosen and tight playing, and spiral winding actions, and its entity is flexible, round, soft, and incessant, liking floating clouds and flowing water, and it could offer warm and harmonious beauty, without cruel fight.

As one kind of traditional Martial Arts of China, Taijiquan stressed three-joint and six-coordination, and the three-joint includes "top", "middle", and "root", and the six-coordination is the coordination of hand, elbow, shoulder, foot, knee, and hip, which is also the embodiment of "harmony".

\subsection{Harmony in the Interior}

For the individual, the "joint" emphasized by Taijiquan means not only the joint of certain parts and some junctures, but also interior three-joint, i.e. "the joint of heart and meaning, the joint of meaning and air, and the joint of air and power", and "the joint of heart and eye, the joint of liver and tendon, the joint of spleen and meat, the joint of lungs and body, and the joint of kidney and bone", and "one joint means the joint of all parts, including five internal organs and all bones (Jiang, 2008, p.126-128)", and in this way, main and collateral channels, air and blood would be harmonized naturally, running smoothly, and internal organs' normal functions could be balanced, and people could be "harmonious" people.

\subsection{Harmony in the Coincidence of the Interior and the Exterior}

Except for interior three-joint and the exterior joint, Taijiquan practitioners also deeply studied the "heart" dominating form, and insisted the opinions combining body with spirit, such as "the coincidence of the interior and the exterior", "the integration of the interior and the exterior", and "the coincidence of form and meanings". From the combination of spirit and body, practitioners deeply understood that "the body should be used by heart, and heart could drive body" based on the opinion that the spirit is the master of the body. The bridge between "heart" and 
"body" is "air", and the harmonious form of Taijiquan between body and meaning is to harmonize the entrails and four limbs, and perfect the healthy body, and the innovation is "to run air by meanings, and air drives forms after expressing meanings". In this way, "four limbs could be driven by heart and air", and the combination of body and heart with "heart volleys with power and all pulses in the body" could be achieved, i.e. the coincidence of spirit and body.

When combining body with heart, Taijiquan practitioners should also centralize immaterial mind, i.e. not only four limbs, but also the spirit should be combined together, and only in this way, the "heart" on the main state could unhurriedly drive bones and fleshes, which could follow the "heart" without protest, achieving the coincidence of spirit and body, and the harmonious beauty of body and heart.

\section{Harmony in Education}

Taijiquan is one of the forms with Chinese traditional culture miniature, and in the times calling for the regression of traditional culture, the education meaning of Taijiquan is more and more important. The education of Taijiquan means not only to perfect individual personality, but also to teach people how to get along with others, the society, and the nature.

\subsection{Harmony in Conducting Oneself}

In China at present, the influence of western culture is more and more serious, and the pragmatism has been the reference point of active action and negative action for many people, and the "cashing mentality" when they do everything. People have gradually given up their principle of conducting themselves, and compromised to material interests and the foam culture begun occur. Facing the economic and practical interests which are difficult to resist, people seem to have no choice, and quickly become the capture of materials, and make their own real and great ideas and targets destroyed, and with it, the personality begins to distort and perish (Yan, 2005, p.63-65).

As the effective operation mode and the representation of harmony culture, Taijiquan sports would compensate the deficiencies of west humanism spirit value. In the exercises of Taijiquan, the moderation is emphasized, and entity exists in empty, and empty exists in entity, and soft exists in hard, and action exists in silence, and Yin and Yang coexist, and practitioners should also be modest and gentle, with dense thinking, and they should like the cobble stones in the sea when handling affairs, and they should never lose their essentials, and achieve the perfect of "neutralization". When the harmony concept enters into people's heart, they could find the life interests, meanings, life values, and spirit orientation in the daily life (Qiu, 2005, p.70-72).

Taijiquan is the extremity of harmony, and the harmony is the balance of Yin and Yang, and the inaction is the only way to realize Taijiquan. Inaction is not the negative action, but the great action. "Tao" is the standard to distinguish "action" and "inaction". The "action" combining with "Tao" could be called as "inaction", and the "action" which could not combining with "Tao" could be called as "action", and all things begin in action, and action includes the good action and the bad action, and the good action contains the ideal of "inaction", orienting to the extreme harmony. Taijiquan begins from visible forms, and then regulates the breath, and when the interior and the exterior go to harmony, and inaction would be achieved, and the personality of the practitioner would be perfected.

\subsection{Harmony with People}

Some people thought that Chinese traditional culture was the emotional culture, which opposes to isolate people from the big system of social interpersonal relationship, and emphasizes that people exist in the society, and pays attention to the harmonious relationship among people. The harmony in Taijiquan is represented in boxing and hands-pushing.

In John Naisbitt's book of "Megatrends", he put forward, "when a new technology is introduced into the society, the human being will certainly produce a kind of reaction to balance it, i.e. a kind of high emotion". High emotion will be centralized and cultured in the society and interpersonal relationship. However, in the day that technology develops so quickly, technology makes people to become more and more estranged and lonely. Suddenly, people would find that, Taijiquan is the high emotion activity to balance the technology, as a kind of sports mode to calm the mind, discipline the temperament, and seek silence in sports. Therefore, to actively organize and extend Taijiquan is a kind of high emotion activity to adapt the modern society and cultivate harmonious interpersonal relationship (Qiu, 2005, p.70-72).

In addition, the actual combat of Taijiquan is hand-pushing, and from brutal fight to "double hands-pushing", Taijiquan could not only realize the evolution from "barbarous" to civilization, but fully embody the moderation ideal and humanistic care. Many attack and defense principles in hands-pushing of Taijiquan such as "win by striking only after the enemy had struck", "give up ones' own views and follow others", "reaching a high of indemnity", "face fast actions by fast actions, and face slow actions by slow actions", and "take the quiet approach, 
and conquer the unyielding with the yielding" could more embody the respects to the opponent in the moral ethics, and it is the wonderful embodiment of traditional martial morality (Guo, 2008, p.33-34).

\subsection{Harmony with the Nature}

"The unity of heaven and humanity" is the essence of the ancient philosophy of China, and "heaven" is the nature. This culture is also integrated into Taijiquan with deep cultural meanings. Therefore, Taijiquan emphasizes the harmony and unity of humanity and nature. Taijiquan practitioners think that humanity is "small Taijiquan", and nature is the "big Taijiquan", and "small Taijiquan" should be harmoniously developed in the "big Taijiquan". Circle and empty are basic characteristics of the nature, and circle means flexible and smooth, and empty means light and free. In Taijiquan, circle and empty are always embodied, so it could embody the harmony of humanity and nature in theory.

For concrete actions, "regulating of breathing" and "six-factor breathing" in the "Hunyuan Piling" of Taijiquan all require practitioners to keep the breathing communication and the information communication between the interior environment with the exterior environment in the states of "without thinking, without concepts, without forms, and without phases". In the exercise of Taijiquan, practitioners should also lead the actions by their consciousness, and live up to "calming the nerves, exercising silently, forgetting environment and themselves, coordination of mental activities and breathing, and breathing naturally", and enter into the nature to achieve the harmonious unity with the nature.

As a general rule, Taijiquan expresses the dominant and implicit natural spirits through "forms", and creates the wonderful state of communicating humanity with nature harmoniously (Lin, 2007, p.21-24). By Taijiquan, practitioners could understand the nature, return the nature, achieve the natural spirit, and follow the natural development.

\section{Harmony in Development}

How many people are exercising Taijiquan in the world? Would the number is one hundred million or two hundred million? Of course, the exact number could not be accounted, but it indicates that Taijiquan is the widest boxing sort in the world at present. From Chen Wangting created Taijiquan to it begun to gradually mature, the whole process is full of harmonious notes.

Taijiquan has five schools at present from its initial Chen's Taijiquan, and though various schools' styles, postures, and technical points are different, and various schools have their own creating and development stories, their own targets and requirements, but they all pursue harmonious concepts, so these schools in Taijiquan could respect each other, and they could ensure their development state in the society gradually because of exterior challenges and rejections. And just because of the principle of "letting the arts have free expression" in various schools of Taijiquan, Taijiquan could present harmonious development state.

Practice is the sole criterion foresting truth, and through hundred years and hundred million people's test, Taijiquan is developing gloriously, which also proves that it could accord with the social development and the efficiency of public body-building. In foreign countries, many scholars proved that Taijiquan exercise could effectively enhance human immunity and improve the functions of various systems, and some one said that it was the best aerobic exercise in the world. In any event, fact is the most powerful proof, and Taijiquan needs not packaging and magnificent appearances, and depending on its own charm, more and more people would participate in the tide of harmonious body-building of Taijiquan.

\section{Conclusions}

With eastern philosophical ideas, Taijiquan is always embodying the harmony of sport, the harmony of education, and the harmony of development, and put the concept of "harmony" into effective operation layer, and it is not only beneficial to the national development, but also construct the harmonious bridge for human beings. Of course, one sports event could not contain all social formations, and could not bring direct economic benefits, but in the correct leading of human being, the value view, and ethics and humanity spirit embodied in Taijiquan, could be useful for human physical and psychological health, and the stability and development of the society.

\section{References}

Guo, Huai. (2008). On the Concept of Harmony in Taijiquan. Boji (Wu Shu Science). No.5(12). p.33-34

Jiang, Juan. (2008). Study on the Harmonious Culture of Taijiquan. Journal of Shenyang Sport University. No.27(3). p.126-128

Lin, Xiaomei, Su, Xin \& Yang, Jianying. (2007). Discussion on Harmonious Ideology and Modern Harmonious Society of Taijiquan. China Sport Science. No.27(11). p.21-24 
Qiu, Pixiang \& Tian, Xuejian. (2005). A Study on the Sense of Worth of General Harmony of Shadowboxing. Journal of Physical Education. No.12(3). p.70-72

Wang, Guozhi, Qiu, Pixiang \& Guo, Huashuai. (2009). Taijiquan: A Typical Note of Feeling and Recognizing Harmony. Journal of Shanghai Physical Education Institute. No.33(1). p.64-67

Yan, Shiliang, et al. (2005). Opportunity and Imagination for Development of Chinese Traditional Wushu. Journal of Anhui Sports Science. No.26(2). p.63-65 